\title{
Impact of psoriasis on serum lipid profile, CRP, ADA: A controlled study of 25 patients
}

\author{
Rita Vora $^{1, *}$, Nishit Surti ${ }^{2}$, Rahul Krishna Kota ${ }^{3}$, Trusha Patel ${ }^{4}$, Khushboo Modasia ${ }^{5}$ \\ ${ }^{1}$ Professor, ${ }^{2}$ Assistant Professor, ${ }^{3}$ Ex-Resident, ${ }^{4}{ }^{\text {rd }}$ Year Resident, ${ }^{5}{ }^{\text {nd }}$ Year Resident, Dept. of Skin \& DVL, Pramukhswami \\ Medical College Gujarat, India \\ *Corresponding Author: \\ Email: ritavv@charutarhealth.org
}

\begin{abstract}
Introduction: Psoriasis is a chronic disorder with certain systemic compounds. It has been associated with increase in morbidity and mortality from cardiovascular events especially in chronic cases.

Aims and Objectives: To compare the levels of CRP, S.ADA and S. Lipid profile among psoriatic patients and controls. Materials and Methods: This is a cross sectional case control study conducted in department of dermatology for a period of 6 months in a teaching institute of Gujarat. Cases included patients of psoriasis attending skin OPD. Controls were taken from health check-up scheme of our hospital with minimal confounding factor. CRP, S.ADA and S. Lipid profile were assessed in both groups.

Statistical Analysis: We applied Unpaired T- test for statistical analysis and p value $<0.05$ was considered significant.

Results: Patient and control group consisted of 25 subjects each. Serum CRP, serum cholesterol and serum TG levels were found to be more significantly elevated in cases when compared to controls with p-value (by unpaired t test) of $0.001,0.026$ and 0.044 respectively. Serum ADA, LDL, VLDL was high among cases when compared to controls but were not statistically significant. $(\mathrm{p}>0.05)$.
\end{abstract}

Conclusion: patients of psoriasis have to be evaluated for risk factors of atherosclerosis like Serum lipid profile, CRP.

Keywords: Psoriasis, CRP, ADA, Lipid profile.

\section{Introduction}

Psoriasis is a chronic inflammatory skin disease that affects $1-3 \%$ of the population. ${ }^{1}$ The etiology of psoriasis is complex. It can be designated as a genetic, systemic, inflammatory, and chronic disorder of skin with certain systemic components. Its prevalence and exacerbations/remissions are affected by multiple environmental, immune hormonal and mental factors besides, alcohol and drug abuse. ${ }^{2}$ Traditionally psoriasis has been considered a dermatologic disease, but the contemporary medical evidence supports that psoriasis is actually a multisystem disease. It has been associated with an increased morbidity and mortality from cardiovascular events, especially those with a severe and long duration of psoriatic skin lesions. ${ }^{2}$ Multiple factors like pro-atherogenic lipoprotein profile which includes hypertriglyceridemia raised plasma concentrations of LDL, and a lowered HDL concentration have been reported to be associated with psoriasis. ${ }^{2}$ There is a complex network of inflammatory and immune cells, cytokines, chemokines and growth factors, all of which interact with one another to initiate a cascade of inflammatory events resulting in T-cell infiltration in the epidermis and dermis. ${ }^{3}$ Recently the concept of "psoriatic march" has been proposed, in which chronic cutaneous inflammation in psoriasis leads to systemic inflammation, which, in conjunction with increased oxidative stress triggers a cascade of events including oxidative stress, dyslipidemia, endothelial dysfunction and insulin resistance which increases the risk of cardiovascular complications in these patients. ${ }^{3}$ C-reactive protein (CRP) is the first protein to be demonstrated as a marker of inflammation and tissue damage. S.ADA (Adenosine deaminase), a main enzyme in purine degradation, is a non-specific marker of T-cell activation. Present study focuses on assessment of levels of Serum lipids, CRP, ADA in patients of chronic plaque psoriasis and compare with the same in controls.

\section{Aims and Objectives}

To compare the level of CRP, S.ADA and S. Lipid profile among psoriatic and non-psoriatic persons. To see whether these markers were significantly associated with psoriasis.

\section{Materials and Methods}

The study was carried out in the department of dermatology in a teaching institute at a tertiary care centre of Gujarat for a period of six months. This was a cross sectional, case control study with total of 25 cases and 25 controls. The study population included all consenting patients of psoriasis visiting dermatology department OPD first time. We excluded psoriatic patient who had history of taking systemic anti psoriatic or hypolipidemic drugs. Detailed history was taken and examination was done. Diagnosis of psoriasis was purely clinical. We had taken control from health check-up scheme in our hospital with minimal confounding factor error. CRP, S.ADA and S. Lipid profile were assessed in both groups. We applied unpaired T-test for statistical analysis and $\mathrm{p}$ value < 0.05 was considered significant 


\section{Results}

Patient and control groups each consisted of 25 (15 male and 10 female). Average age of cases was 40 years while that of controls was 42 years. Duration of disease was $<1$ year in 10 patients while it was $>1$ year in the remaining patients (15) with the average duration of disease 17 months.

History of smoking was present in 7 cases and 8 controls while history of alcohol intake was present in 7 cases and 8 controls.

Serum CRP level is elevated in $16(64 \%)$ cases of psoriasis out of total 25 cases and in $7(28 \%)$ controls (Table 1). Serum ADA is elevated in $5(20 \%)$ cases and $1(4 \%)$ controls. Serum cholesterol is elevated in 15 $(60 \%)$ cases and $9(36 \%)$ controls. Serum TG (triglycerides) was elevated in $17(68 \%)$ cases and
$7(28 \%)$ controls. Serum HDL (High density lipoprotein) was not found to be elevated in cases while it was elevated in $1(4 \%)$ control. Serum LDL (low density lipoprotein) is elevated in $12(48 \%)$ cases and $6(24 \%)$ controls while serum VLDL (very low density lipoprotein) is elevated in 9(36\%) cases and $5(20 \%)$ controls. (Table 1)

Serum CRP, serum cholesterol and serum TG levels were found to be more significantly elevated in cases when compared to controls with p-value (by unpaired $t$ test) of $0.001,0.026$ and 0.044 respectively. Serum ADA, LDL, VLDL was high among cases when compared to controls but were not statistically significant. $(\mathrm{p}>0.05)$ (Table 1)

Table 1: Serum lipid profile, serum ADA, serum CRP levels in psoriatic patients and controls

\begin{tabular}{|c|c|c|c|c|}
\hline Parameters & Levels & $\begin{array}{c}\text { Psoriasis } \\
\text { (Cases) }\end{array}$ & $\begin{array}{c}\text { No Psoriasis } \\
\text { (controls) }\end{array}$ & $P$ value \\
\hline \multirow[t]{2}{*}{ S.CRP(6) } & Elevated & $16(64 \%)$ & $7(28 \%)$ & \multirow[t]{2}{*}{0.001} \\
\hline & Normal & 9 & 18 & \\
\hline \multirow[t]{2}{*}{ S.ADA(30) } & Elevated & $5(20 \%)$ & $1(4 \%)$ & \multirow[t]{2}{*}{0.177} \\
\hline & Normal & 20 & 24 & \\
\hline \multirow[t]{2}{*}{ S.Cholesterol(200) } & Elevated & $15(60 \%)$ & $9(36 \%)$ & \multirow[t]{2}{*}{0.026} \\
\hline & Normal & 10 & 16 & \\
\hline \multirow[t]{2}{*}{ S.TG(170) } & Elevated & $17(68 \%)$ & $7(28 \%)$ & \multirow[t]{2}{*}{0.044} \\
\hline & Normal & 8 & 18 & \\
\hline \multirow[t]{2}{*}{ S.HDL(68) } & Elevated & $0(0 \%)$ & $1(4 \%)$ & \multirow[t]{2}{*}{0.277} \\
\hline & Normal & 25 & 24 & \\
\hline \multirow[t]{2}{*}{ S.LDL(130) } & Elevated & $12(48 \%)$ & $6(24 \%)$ & \multirow[t]{2}{*}{0.474} \\
\hline & Normal & 13 & 19 & \\
\hline \multirow[t]{2}{*}{ S.VLDL(38) } & Elevated & $9(36 \%)$ & $5(20 \%)$ & \multirow[t]{2}{*}{0.515} \\
\hline & Normal & 16 & 20 & \\
\hline
\end{tabular}

\section{Discussion}

Psoriasis is an immunologically mediated disease caused by activation of T-lymphocyte that elaborates a Th1 type of immune response. Psoriasis is a chronic inflammatory dermatological condition characterized by increased $\mathrm{T}^{-}$-helper- 1 and $\mathrm{T}$-helper- 17 cell activity. ${ }^{2}$ It has been suggested that psoriasis, like atherosclerosis, could have autoimmunity to play a role in its pathogenesis. ${ }^{2}$ The cytokines implicated in psoriases such as $\mathrm{IL}^{-} 6$, IL- 8 , INF- $\gamma$, IL- 1 , and IL-17 are also implicated in the generation of pro- $^{-}$atheromatous abnormalities. ${ }^{2}$

Serum adenosine deaminase (S. ADA) is an enzyme involved in purine metabolism. It is needed for the breakdown of adenosine from food and for the turnover of nucleic acids in tissues. It functions in development and maintenance of the body's immune system. Its isoform ADA2 is predominantly found in human blood plasma and is found to have increased in diseases associated with immune system. ADA enzyme activity is a non specific marker of T-cell activation.
Cytokines released by both T-cell and keratinocytes mediate keratinocyte proliferation in psoriasis. ${ }^{4}$ In our study, serum ADA is elevated in 5(20\%) cases and $1(4 \%)$ controls with $\mathrm{p}$ value of 0.177 whereas in a study conducted by Bukulmez G et al, Serum ADA level was significantly elevated in patients with psoriasis compared to healthy subjects. ${ }^{5}$ A study showed that elevated ADA activity reflects accelerated salvage pathway of nucleic acid metabolism associated with hyperproliferative status of epidermis in psoriasis. This study also suggested that increased ADA activity is associated with severity of psoriasis. ${ }^{4}$

$\mathrm{C}$ reactive protein (CRP) is a Pentameric protein found in blood plasma. CRP is an acute phase protein and is increased in all kinds of inflammatory and tissue damage processes. CRP has been suggested to be a marker of inflammation in psoriasis. ${ }^{6}$ In our study, serum CRP level is elevated in $16(64 \%)$ cases of psoriasis out of total 25 cases and in $7(28 \%)$ controls with p-value to be 0.001 . Two studies by Vanizor et al. have shown that patients with psoriasis have significantly high baseline levels of CRP compared 
with healthy controls. ${ }^{7,8}$ Malbris et al. noted that patients with psoriasis had higher levels of CRP compared with controls, with a positive correlation between CRP and total plasma cholesterol. ${ }^{9}$ Similar results were observed by Rocha-Periera et al. ${ }^{10}$ In a study evaluating inflammatory markers in patients with mild or severe psoriasis. Several studies also showed correlation between level of CRP and severity of proriasis. ${ }^{11-14}$

A study has showed that acute phase proteins like CRP is an inflammatory marker increased in serum of psoriatic patient. ${ }^{6}$ It is reported that CRP has its relation with cytokines which are responsible for inflammatory changes in skin of psoriasis patients. ${ }^{6}$

Atherogenic dyslipidemia comprises a triad of increase in the small, dense low density lipoprotein cholesterol (LDL-C), decreased high density lipoprotein cholesterol (HDL-C), and increased triglycerides (TGs) in the blood. ${ }^{2}$ Lea et al reported increased density of serum lipids in psoriatic patients around 60 years ago. ${ }^{15}$ The most important role of HDL particle is reverse cholesterol transport. Modified HDL particles in atherosclerotic plaques stimulates cholesterol efflux from foam cells, endothelium dependent vasoreactivity and antioxidative activity and also generates a pro atherogenic species that inhibits nitric oxide synthesis in endothelial cells. ${ }^{16}$ It is reported that macrophages activated by engulfing low density lipoprotein (LDL) immune complexes release large quantities of tumor necrosis factor (TNF) -alpha and IL-1 $\beta .{ }^{16}$ Cytokine driven inflammation and tissue destruction is a common theme of chronic inflammatory diseases such as psoriasis and atherosclerosis. ${ }^{16}$ It is understood that predisposition to vascular obstructive diseases is due to the intensity of psoriasis disease.

In our study, serum cholesterol is elevated in $15(60 \%)$ cases and $9(36 \%)$ controls. Serum TG was elevated in $17(68 \%)$ cases and $7(28 \%)$ controls. Serum HDL was not found to be elevated in cases while it was elevated in $1(4 \%)$ control. Serum LDL is elevated in $12(48 \%)$ cases and $6(24 \%)$ controls while serum VLDL is elevated in $9(36 \%)$ cases and $5(20 \%)$ controls. Serum cholesterol and serum TG levels were found to be more significant in cases compared to controls with p-value (by unpaired $t$ test) to be 0.026 and 0.044 respectively. Dreiher et al found a significant increase in lipid levels among psoriasis patients than in controls (P < 0.001). ${ }^{17}$ Shapiro et al found that psoriasis was associated hyperlipidemia, but was not associated with an increase in LDL level. ${ }^{18}$ Cohen et al have found that psoriasis is associated with dyslipidemia. ${ }^{19}$ In Metta et al study, there was significantly high levels of TC, LDL, VLDL, and TG in psoriasis patients than controls while there was no change in the levels of HDL. ${ }^{20}$ Similar findings were reported by Lateef et al. ${ }^{21}$ Mallbris et al., showed there was higher total cholesterol, VLDL and HDL levels corresponding to normal control group but the was significant for only HDL while in our study, there was no raise of HDL levels in psoriatic patients. ${ }^{9}$ There was significantly raised levels of TC, TGA, LDL in patients of psoriasis when compared to controls in study by Javidi et al. ${ }^{16}$ Piskin in his study showed serum total and LDL cholesterol levels to be significantly higher than that of control group. ${ }^{22}$ Abnormal lipoprotein metabolism may be related to the high incidence of atherosclerosis in psoriasis. ${ }^{23}$ Hypertriglyceridemia secondary to VLDL elevation is associated with both procoagulant and prothrombotic factors in the blood. VLDL mediated platelet adhesion may play an important role in atherosclerosis. Furthermore, VLDL remnants are susceptible to retention within the arterial intima, thereby promoting atherosclerotic plaques growth. A study done by Mallbris et al., which supports the notion that psoriasis could be associated with lipid abnormalities at the onset of the skin disease and lipid abnormalities in psoriasis may be genetically determined rather than acquired. ${ }^{9}$

\section{Conclusion}

It is better to screen every patient of psoriasis for risk factors of cardiovascular disease like Serum CRP, serum lipid level particularly cholesterol, triglycerides to prevent atherosclerosis and its complications.

\section{Limitation}

It is a very small scale study. To substantiate our findings, multi centic trials with larger number of patients are required.

\section{References}

1. Nisa N, Qazi AM. Prevalence of metabolic syndrome in patients with psoriasis. Indian J of Dermatol Venereol Leprol. 2010;76:662-5.

2. Shenoy C, Shenoy MM, Shantaram M, Vishal B, Pinto M, Krishna S. Atherogenic lipid profile in psoriasis: Correlation with severity and duration of the disease. $J$ Obes Metab Res. 2015;2:217-20.

3. Sunitha S, Rajappa M, Thappa DM, Chandrashekar L, Munisamy M, Revathy G, et al. Comprehensive lipid tetrad index, atherogenic index and lipid peroxidation: Surrogate markers for increased cardiovascular risk in psoriasis. Indian J Dermatol Venereol Leprol. 2015;81:464-71.

4. Erbagei Z, Erbgeis AB, Koyliioglu O, Tuncel AA. Serum adenosine deaminase activity in monitoring disease activity and response to therapy in psoriasis, Acta medica (Hrudec Kralov). 2006;49(2):101-4.

5. Bukulmez G, Akan T, Ciliv G University Faculty of Medicine, Department of Dermatology, Ankara, Turkey 06100, USA. bukulmez@hacettepe.edu.tr. European Journal of Dermatology: EJD. 2000;10(4):274-276.

6. Gerkowitz A, Pietrzk A, Szepietowski JC, Radej S, Chodorowska G. Biochemical marker of psoriasis as a metabolic disease. Folia Histochemista et Cytobiologica. 2012;50(2):155-70.

7. Vanizor Kural B, Orem A, Cimşit G, Yandi YE, Calapoglu M. Evaluation of the atherogenic tendency of lipids and lipoprotein content and their relationships with 
oxidant- antioxidant system in patients with psoriasis. Clin Chim Acta. 2003;328:71-82.

8. Vanizor Kural B, Orem A, Cimşit G, Uydu HA, Yandi YE, Alver A. Plasma homocysteine and its relationships with atherothrombotic markers in psoriatic patients. Clin Chim Acta. 2003;332:23-30.

9. Mallbris L, Granath F, Hamsten A, Ståhle M. Psoriasis is associated with lipid abnormalities at the onset of skin disease. J Am Acad Dermatol. 2006;54:614-21.

10. Rocha- Pereira P, Santos-Silva A, Rebelo I, Figueiredo A, Quintanilha A, Teixeira F. The inflammatory response in mild and in severe psoriasis. Br J Dermatol. 2004; 150:917-28.

11. Vadakayil AR, Dandekeri S, Kambil SM, Ali NM. Role of C-reactive protein as a marker of disease severity and cardiovascular risk in patients with psoriasis. Indian Dermatol Online J. 2015;6:322-5.

12. Coimbra S, Oliveira H, Reis F, Belo L, Rocha S, Quintanilha A, et al. C- reactive protein and leucocyte activation in psoriasis vulgaris according to severity and therapy. J Eur Acad Dermatol Venereol. 2010;24(7):78996.

13. Kimball AB, Wu Y. Cardiovascular disease and classic cardiovascular risk factors in patients with psoriasis. Int $J$ Dermatol. 2009;48:1147-56.

14. Coimbra S, Oliveira H, Reis F, Belo L, Rocha S, Quintanilha A, et al. Circulating adipokine levels in portugese patients with psoriasis vulgaris according to body mass index, severity and therapy. J Eur Acad Dermatol Venereol. 2010;24:1386-94.

15. Lea WA Jr, Cornish HH, Black WD. Studies on serum lipids protein and lipoproteins in psoriasis. J Invest Dermatol. 1958;30:181-5.

16. Javidi Z, Meibodi N T, Nahidi Y. Serum lipids abnormalities and psoriasis. Indian J Dermatol. 2007;52(2):89-92.
17. Dreiher J, Weitzman D, Davidovici B, Shapiro J, Cohen AD. Psoriasis and dyslipidaemia: A population- based study. Acta Derm Venereol. 2008;88:561-5.

18. Shapiro J, Cohen AD, Weitzman D, Tal R, David M. Psoriasis and cardiovascular risk factors: A case- control study on inpatients comparing psoriasis to dermatitis. $J$ Am Acad Dermatol. 2012;66:252-8.

19. Cohen AD, Dreiher J, Shapiro Y, Vidavsky L, Vardy DA, Davidovici B, et al. Psoriasis and diabetes: A population- based cross- sectional study. J Eur Acad Dermatol Venereol. 2008;22:585-9.

20. Metta S, Kumar MA, Basalingappa RD, Uppala S, Mohanty S. Circulatory markers of oxidative stress and dyslipidemia in male patients of chronic plaque psoriasis. Int J Med Public Health. 2015;5:208-12.

21. Lateef A, Mohanty S, Reddy D, Rao P. Atherogenic index and proteinfractions in Psoriasis. Int J Med Pubic Health. 2011;1:25-30.

22. Piskin S, Gurkok F, Ekuklu G, Senol M. Serum lipid levels in Psoriasis. Yonsei Med J. 2003;44:24-6.

23. Seishima M, Seishima M, Mori S, Noma A. Serum lipid and apolipoprotein levels in patients with psoriasis. $\mathrm{Br} J$ Dermatol. 1994;130:738-42.

How to cite this article: Vora R, Surti N, Kota RK, Patel T, Modasia K. Impact of psoriasis on serum lipid profile, CRP, ADA: A controlled study of 25 patients. Ind J Clin Exp Dermatol. 2018;4(3):246-249. 